\title{
Cinética de la degradación y estabilidad del ácido ascórbico en la pulpa de copoazu (Theobroma grandiflorum)
}

Kinetics of the degradation and stability of ascorbic acid in the pulp of copoazu (Theobroma grandiflorum)

Cuse Quispe, Jaime ${ }^{1[0000-0001-6424-4934]}$; Cutipa Chávez, Lastenia ${ }^{2[0000-0003-}$ 3341-1095] y Lima Pimentel, Víctor Hugo ${ }^{2[0000-0003-4126-9543]}$

${ }^{1}$ Universidad Nacional Amazónica de Madre de Dios ${ }^{2}$ Universidad Nacional de San Martin, Tarapoto, Perú jcuseqeunsm.edu.pe

Resumen. La investigación fue realizada en Madre de Dios y tuvo como objetivo determinar la cinética de degradación y estabilidad del ácido ascórbico en la pulpa de copoazú (Theobroma grandiflorum) con y sin tratamiento térmico durante su almacenamiento por 80 días a diferentes temperaturas $\left(5^{\circ} \mathrm{C},-5^{\circ} \mathrm{C}-15^{\circ} \mathrm{C}\right)$. Los materiales que se utilizaron fueron: Copoazú (Theobroma grandiflorum), Materiales de acondicionamiento como tina y balanza digital, Materiales de laboratorio como pipetas y buretas, Equipos como la pulpeadora, Reactivos 2,6-diclorofenol indofenol. En cuanto a los métodos se utilizó el método estándar de la AOAC modificado por BENASSI \& ANTUNES, (1988). En cuanto a los resultados se evidencio que existe diferencia estadísticamente significativa entre un tratamiento y otro con respecto al nivel de temperatura de almacenamiento de la pulpa de copoazú ( $\mathrm{P}$ va-lor $<0.05$ ), con un nivel de confianza del $95 \%$, Concluyéndose que el ácido ascórbico sigue una reacción de deterioro de orden uno tanto para las muestras con y sin tratamiento térmico. A través de la ecuación de Arrhenius fue posible determinar la energía de activación referente a la degradación del ácido ascórbico durante su almacenamiento; para el ajuste con el modelo de cinética de primer orden se encontró una EaTT =25,10Kcal $/ \mathrm{mol}$ y EaSTT = $10,5098 \mathrm{Kcal} / \mathrm{mol}$.

Citar como: Cuse Quispe, J., Lima Pimentel, V. H. ., \& Cutipa Chávez, L. . . (2021). Cinética de la degradación y estabilidad del ácido ascórbico en la pulpa de copoazu

(Theobroma grandiflorum). Revista Agrotecnológica Amazónica, 1(1), 4-19. https://doi.org/10.51252/ra a.v1i1.113

Recibido: 15/11/2020

Revisado: $15 / 12 / 2020$

Publicado: 31/01/2021

\section{Palabras clave: ácido ascórbico, cinética, pulpa, theobroma grandiflorum}

Abstract. The research was carried out in Madre de Dios and its objective was to: Determine the degradation kinetics and stability of ascorbic acid in copoazú pulp (Theobroma grandiflorum) with and without heat treatment during storage for 80 days at different temperatures $\left(5^{\circ} \mathrm{C}\right),,-5^{\circ} \mathrm{C}-15^{\circ}$ C). The materials used were: Copoazú (Theobroma grandiflorum), Conditioning materials such as vat and digital balance, Laboratory materials such as pipettes and burettes, Equipment such as the Pulper, Reagents such as 2,6-diclorofenol indofenol. Regarding the methods, the standard AOAC method modified by BENASSI \& ANTUNES, (1988) was used. Regarding the results, it was evidenced that there is a statistically significant difference between one treatment and another with respect to the storage temperature level of the copoazú pulp ( $\mathrm{P}$ value $<0.05$ ), with a confidence level of $95 \%$, Concluding that ascorbic acid follows a deterioration reaction of order one both for the samples with and without heat treatment. Through the Arrhenius equation it was possible to determine the activation energy related to the degradation of ascorbic acid during its storage; For the adjustment with the first order kinetic model, an $\mathrm{EaTT}=25.10 \mathrm{Kcal} / \mathrm{mol}$ and EaSTT $=10.5098 \mathrm{Kcal} / \mathrm{mol}$ was found .

Keywords: ascorbic acid, kinetics, pulp, theobroma grandiflorum 


\section{Introducción}

Uno de los procesos de conservación de alimentos más importantes es el almacenamiento a bajas temperaturas, no solo por los efectos deseables que se obtienen, sino por la calidad organoléptica final del producto. Se ha estudiado que el almacenamiento de alimentos a bajas temperaturas inhibe la actividad microbiana, enzimática y preserva los nutrientes contenidos en la matriz alimentaria.

El copoazu es un producto con un alto contenido de ácido ascórbico $(58,22 \mathrm{mg} / 100 \mathrm{~g})$, resultado semejante a lo reportado por Martin (2013), quien en su estudio encontró 56,16 mg ácido ascórbico/100 g. de muestra.

En la constante de velocidad está involucrada principalmente la afectación de temperatura, el aumento en la temperatura produce un aumento en la velocidad de la mayor parte de las reacciones, alterando las concentraciones de ácido ascórbico (Mendoza y Quiñones, 2005)

El copoazú presenta alto contenido de pectina, comparable al de la manzana, fibra dietética soluble que, según algunos trabajos, han demostrado reducción de los niveles séricos de colesterol y triglicéridos en ratas y humanos (Fietz \& Salgado, 1999; Arjmandi et al. (1992).

Melgarejo (2006), indica que el copoazú es una planta leñosa, arbórea de ciclo perenne, de crecimiento erecto y semirrecto, de forma cilíndrica, el árbol puede crecer hasta $18 \mathrm{~m}$. en estado silvestre, cultivado alcanza una altura entre $5 \mathrm{~m}$. a $8 \mathrm{~m}$. La forma de las semillas es elípticaaplanada (Velez, 1991). El fruto puede contener de 20 a 50 semillas, pero existen plantas que producen frutos sin semillas (Lim, 2012).

El rendimiento de pulpa de copoazú reportado por Venturieri (1993) tiene un rendimiento de $38,5 \%$ y Arizaleta et al., (2015) con 32,79\% en masa fresca.

El ácido ascórbico es considerado un elemento altamente termo sensible, a diferencia de otras vitaminas cuando es sometido a procesamiento térmico, este llega a perder hasta el $100 \%$ de su concentración si no llega a ser tratado adecuadamente (Santos y Silva, 2008). Por tal motivo la concentración residual de ácido ascórbico es considerado como un indicador de calidad nutricional durante el procesamiento y almacenamiento de alimentos.

La vitamina $\mathrm{C}$ se destruye térmicamente vía anaeróbica no oxidativa, de menor importancia, que alcanza su máximo a pH 4 como ocurre en jugos de limón y concentrados de naranja, en los que el oscurecimiento va acompañado de la formación de furfural y cuya cuantificación refleja el daño térmico (Badui, 2013).

La predicción de la cantidad de pérdida de ácido ascórbico requiere conocer la cinética de degradación, siendo la constante de degradación específica para cada producto y se determina Citar como: Cuse Quispe, J., Lima Pimentel, V. H. ., \& Cutipa Chávez, L. . (2021). Cinética de la degradación y estabilidad del ácido ascórbico en la pulpa de copoazu (Theobroma grandiflorum). Revista Agrotecnológica Amazónica, 1(1), 4-19. https://doi.org/10.51252/raa.v111.113 
experimentalmente. Se han realizado estudios para determinar la cinética de degradación en vitaminas, y se establece que estas reacciones siguen una ecuación de primer orden, cuya contante de degradación está regida por el modelo de Arrhenius.

Según Azeredo (2012).al someter la pulpa a un proceso térmico se inactivan las enzimas presentes en la pulpa in natura, deteniendo el proceso de degradación y fermentación. De igual forma, a bajas temperaturas pueden ocasionar perdida del ácido ascórbico (Tavares:1998), y las bajas temperaturas retrasan la actividad enzimática y el crecimiento microbiano (Gonzales, 2010);

Durante el proceso térmico aplicado, se elimina agua a través de la evaporación, logrando una mayor concentración de los sólidos solubles, reflejándose en un aumento en la lectura de ${ }^{\circ}$ Brix (Castellano: 2016).

El ácido ascórbico en muestras almacenadas a $5^{\circ} \mathrm{C}$ reportó una influencia significativa de la temperatura de almacenamiento, similar a lo reportado por Matos y Chuquilín, (2010).

Se trabajó con tratamiento térmico a $90^{\circ} \mathrm{C}$ por $60 \mathrm{seg}$. y sin tratamiento térmico, y temperaturas de almacenamiento $\left(5^{\circ} \mathrm{C},-5^{\circ} \mathrm{C}\right.$ y $\left.-15^{\circ} \mathrm{C}\right)$; para determinar las propiedades fisicoquímicas (ácido ascórbico, $\mathrm{pH}$, acidez total titulable y solidos totales) en la pulpa de copoazú (Theobroma grandiflorum); planteándose si la cinética de degradación y estabilidad del ácido ascórbico en la pulpa de copoazú (Theobroma grandiflorum) no es influenciada por la temperatura de tratamiento térmico y almacenamiento. Utilizando para cada tratamiento $10 \mathrm{Kg}$ de pulpa de un total de $100 \mathrm{Kg}$ de frutos de copoazú fisiológicamente maduros.

El objetivo de la investigación fue Determinar la cinética de degradación y estabilidad del ácido ascórbico en la pulpa de copoazú (Theobroma grandiflorum) con y sin tratamiento térmico durante su almacenamiento por 80 días a diferentes temperaturas $\left(5^{\circ} \mathrm{C},-5^{\circ} \mathrm{C}-15^{\circ} \mathrm{C}\right)$ y entre los resultados más resaltantes se observó que existe diferencia estadísticamente significativa entre un tratamiento y otro con respecto al nivel de temperatura de almacenamiento de la pulpa de copoazú (P valor<0.05), con un nivel de confianza del 95\%, lo que nos indica que los diferentes tratamientos influyen significativamente en al menos un tratamiento en el contenido de ácido ascórbico presente en la pulpa de copoazú tratada térmicamente.

\section{Materiales y Métodos}

La población estuvo constituida por $100 \mathrm{~kg}$ de frutos de copoazú (Theobroma grandiflorum) fisiológicamente maduros, de los cuales se espera obtener un rendimiento promedio del $38 \%$ de pulpa.

Citar como: Cuse Quispe, J., Lima Pimentel, V. H. ., \& Cutipa Chávez, L. . (2021). Cinética de la degradación y estabilidad del ácido ascórbico en la pulpa de copoazu (Theobroma grandiflorum). Revista Agrotecnológica Amazónica, 1(1), 4-19. https://doi.org/10.51252/raa.v111.113 
La cantidad de muestra a utilizar estuvo constituida por $10 \mathrm{~kg}$ de pulpa de copoazú (Theobroma grandiflorum) distribuidos en bolsas de polietileno de alta densidad con $100 \mathrm{~g}$. de pulpa necesarios para realizar los análisis físico químicos.

\subsection{Métodos}

\section{Determinación de ácido ascórbico.}

Para la determinación del contenido de Ácido ascórbico se utilizó el método estándar de la AOAC modificado por BENASSI \& ANTUNES, (1988) basada en que el ácido ascórbico reduce el indicador DCFI, a una solución incolora, la cual, al llegar al punto final de titulación, se torna en un color rosa pálido en medio ácido.

Solución de Acido oxálico al 2\%: Se debe guardar en envase de vidrio oscuro en refrigeración para su conservación.

Solución 2,6-diclorofenol indofenol al 0,01\%: Debe ser almacenada en envase de vidrio oscuro y en refrigeración. Pesar en balanza analítica 100 mg de DCFI e 210 mg de bicarbonato de sodio, Diluir en $50 \mathrm{ml}$ de agua destilada caliente el DCFI y completar en balón volumétrico de 1 litro, juntamente con el bicarbonato de sodio. Estandarizar con solución estándar de ácido ascórbico.

Ácido ascórbico: Se prepara una solución más concentrada y de esta se toma un alícuota y prepara la solución. Para la solución padrón o estándar se pesa exactamente $125 \mathrm{mg}$ de ácido ascórbico en balanza analítica, diluir con ácido oxálico en balón volumétrico de $50 \mathrm{ml}$.

Estandarización de la solución DCFI: tomar $1 \mathrm{ml}$ de solución estándar de ácido ascórbico, completar el volumen en un balón de $25 \mathrm{ml}$ con ácido oxálico $2 \%$, tomar un alícuota de $10 \mathrm{ml}$ y titular con solución estándar DCFI (duplicado).

Procedimiento: pesar $25 \mathrm{~g}$ de muestra, mezclar con $50 \mathrm{~g}$ de solución de ácido oxálico, del extracto obtenido retirar un alícuota de $10 \mathrm{~g}$, diluir a $50 \mathrm{ml}$ en balón volumétrico y filtrar. Retirar un alícuota de $10 \mathrm{ml}$ de la solución y titular con DCFI (punto de viraje-aparecimiento color rosa). El contenido de ácido ascórbico de las muestras se calcula utilizando la siguiente ecuación:

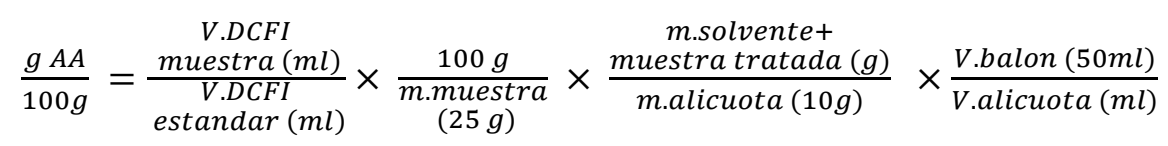

Dónde:

V. DCFI = Volumen del 2, 6 Diclorofenol indofenol

$\mathrm{m} \quad=$ Masa

Citar como: Cuse Quispe, J., Lima Pimentel, V. H. ., \& Cutipa Chávez, L. . . (2021). Cinética de la degradación y estabilidad del ácido ascórbico en la pulpa de copoazu (Theobroma grandiflorum). Revista Agrotecnológica Amazónica, 1(1), 4-19. https://doi.org/10.51252/raa.v1i1.113 
V. balón = Volumen del balón

V. alícuota $=$ Volumen del alícuota

\section{Acidez (\%)}

Se realiza titulación mediante la valoración de la muestra con $\mathrm{NaOH} 0.1 \mathrm{~N}$ y fenolftaleína como indicador expresado en porcentaje de ácido cítrico con peso equivalente a 64.04. AOAC 942.15 (2005).

\section{Solidos solubles $\left({ }^{\circ}\right.$ Brix $)$}

Se determinó mediante refractómetro digital a una temperatura de $20^{\circ} \mathrm{C}$. AOAC 931.12 (2005).

\section{Determinación de pH}

Se determinó mediante la valoración potenciométrica con un pH-metro. AOAC 981.12 (2005)

\subsection{Metodología experimental}

La metodología consistió en evaluar el contenido de ácido ascórbico en la pulpa de copoazú en cada etapa de los procesos normalmente utilizados para su obtención, con y sin tratamiento térmico y su almacenamiento a 3 temperaturas durante 80 días; luego se determinaron los parámetros cinéticos de la degradación del ácido ascórbico en la pulpa de copoazú.

\section{a. Descripción del flujo de proceso de obtención de pulpa de copoazú.}

\section{Materia prima}

Los frutos de copoazú fueron colectados totalmente maduros fisiológicamente. Estos son trasladados a la Planta Piloto de Frutas de la Universidad Nacional Amazónica de Madre de Dios.

\section{Recepción}

Se verificó superficialmente la materia prima, separando los que presentes rajaduras o deterioro.

\section{Pesado}

Se efectuó el pesado de los frutos para determinar rendimiento.

\section{Lavado}

Se eliminó las partículas que se encuentran en la superficie del fruto como tierra, polvo y/o partículas extrañas. Esto se realiza con agua potable y una escobilla de cerdas plásticas.

\section{Selección}

Se verificó que el estado de los frutos reúna las características establecidas, se eliminan los frutos que aun presentan daños o inadecuados para el procesamiento, los que pudieron no ser verificados durante su recepción.

Citar como: Cuse Quispe, J., Lima Pimentel, V. H. ., \& Cutipa Chávez, L. . . (2021). Cinética de la degradación y estabilidad del ácido ascórbico en la pulpa de copoazu (Theobroma grandiflorum). Revista Agrotecnológica Amazónica, 1(1), 4-19. https://doi.org/10.51252/raa.v1i1.113 


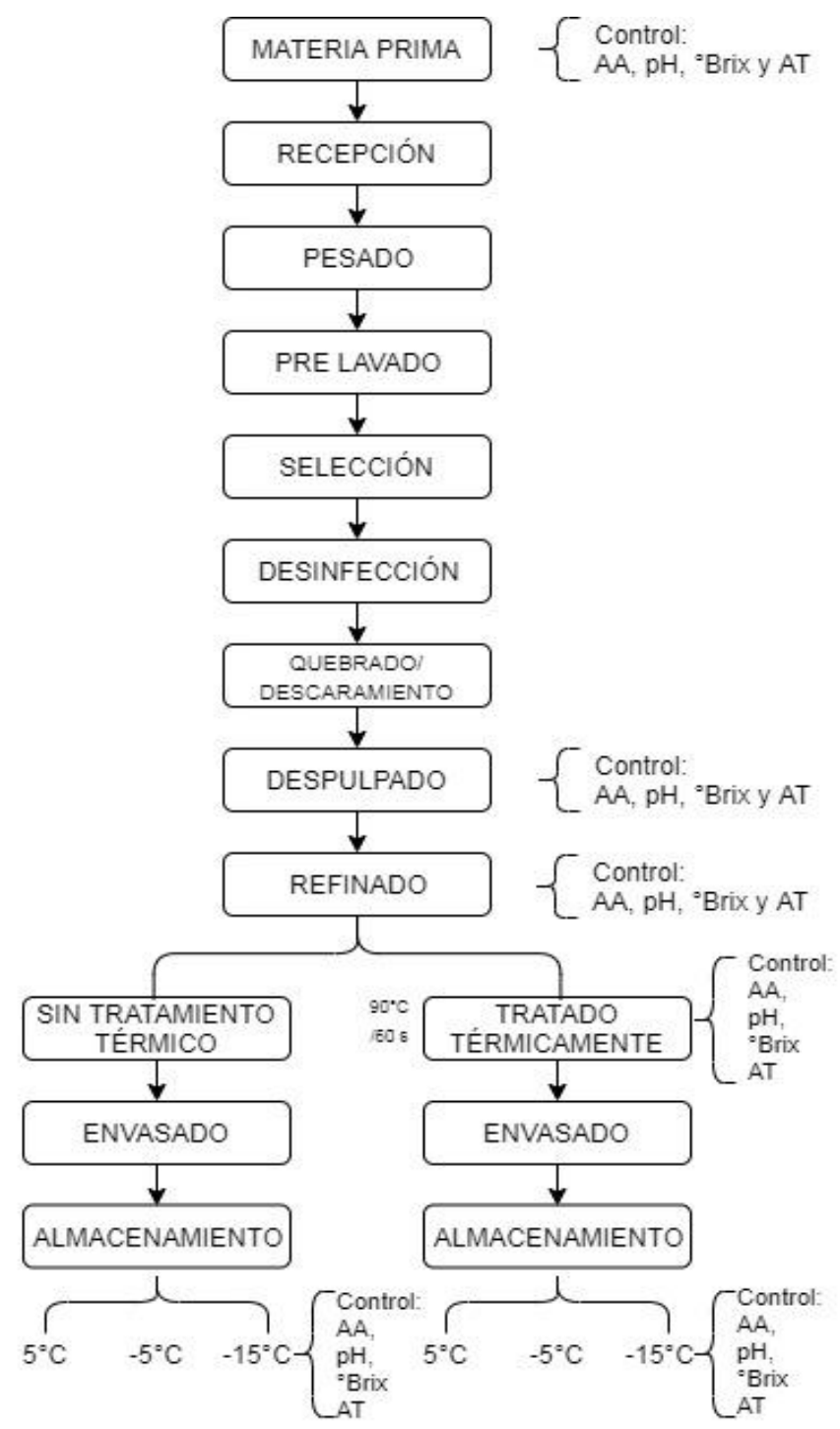

Figura 1: Diagrama de flujo del proceso de obtención de pulpa de copoazú y tratamiento

\section{Desinfección}

Se realizó con una solución de agua clorada $100 \mathrm{mg}$. L-1 por inmersión durante 5 minutos para inactivar la carga microbiana presente en el fruto.

\section{Quebrado/descascarado}

Se extrajo la pulpa junto a la semilla del fruto mediante un martillo de goma, el cual fracciona la cáscara permitiendo su remoción; así mismo se va separando las fibras, para luego llevarlo a la despulpadora.

\section{Despulpado}

Se realizó en una despulpadora con una malla de orificio de 2,9 mm de diámetro, esta operación nos permite separar las semillas y fibras que aun pudiesen permanecer junto a la pulpa.

\section{Refinado}

Citar como: Cuse Quispe, J., Lima Pimentel, V. H. ., \& Cutipa Chávez, L. . (2021). Cinética de la degradación y estabilidad del ácido ascórbico en la pulpa de copoazu (Theobroma grandiflorum). Revista Agrotecnológica Amazónica, 1(1), 4-19. https://doi.org/10.51252/raa.v1i1.113 
Una vez obtenida la pulpa de copoazú, se sometió a refinamiento en licuadora comercial durante 3 minutos para obtener un producto homogéneo.

\section{Pasteurizado}

Obtenida la pulpa refinada de copoazú, esta es divida en dos grupos: un grupo sin tratamiento térmico; y el otro sometido a pasteurización. La pulpa es pasteurizada a $90^{\circ} \mathrm{C}\left( \pm 2^{\circ} \mathrm{C}\right)$ durante 60 segundos para inactivar la acción enzimática de la pulpa.

\section{Envasado}

El envasado se realizó en bolsas de alta densidad con aditivo EVA (resistente a bajas temperaturas) en cantidades de $100 \mathrm{~g}$.

\section{Almacenamiento}

Los grupos de muestras fueron almacenados a tres temperaturas $\left(5^{\circ} \mathrm{C},-5^{\circ} \mathrm{C}\right.$ y $\left.-15^{\circ} \mathrm{C}\right)$ por un periodo de 80 días.

\section{b. Parámetros cinéticos de degradación del ácido ascórbico en la pulpa de copoazú}

Para obtener los valores de los parámetros cinéticos en la pulpa de copoazú se utilizó el método gráfico.

\section{Determinación de la constante de velocidad de reacción de degradación (K)}

Este valor de la constante $\mathrm{K}$ se obtuvo por cada tratamiento mediante el valor de la pendiente de la regresión lineal del logaritmo de la concentración del ácido ascórbico en función del tiempo.

$\log C=\log C_{0}-\frac{K}{2,303} * \theta$

\section{Determinación de la Energía de Activación (Ea)}

Para determinar la Energía de activación, se evaluó la influencia de la temperatura sobre la contante de velocidad de reacción (K) en función del inverso de la temperatura absoluta correspondiente, para luego aplicar regresión lineal.

$\log K=\log K_{0}-\frac{E a}{2,303 R} * \frac{1}{T}$

\section{Tratamientos de los datos}

Para el análisis estadístico se efectuó un diseño estadístico completamente al azar (DCA) para la muestra con y sin tratamiento térmicamente a las mismas condiciones. Los resultados fueron sometidos a análisis de varianza ANOVA para comparar las medias con nivel de confianza del $95 \%$.

Para el análisis del presente estudio se utilizó el software desing-xpert y OriginPro 8.

Citar como: Cuse Quispe, J., Lima Pimentel, V. H. ., \& Cutipa Chávez, L. . . (2021). Cinética de la degradación y estabilidad del ácido ascórbico en la pulpa de copoazu (Theobroma grandiflorum). Revista Agrotecnológica Amazónica, 1(1), 4-19. https://doi.org/10.51252/raa.v111.113 
Tabla 1

Diseño experimental de la investigación.

\begin{tabular}{|c|c|c|c|}
\hline Materia prima & $\begin{array}{c}\text { Tratamiento } \\
\text { térmico }\end{array}$ & $\begin{array}{c}\text { Temperatura de } \\
\text { almacenamiento }\end{array}$ & Observaciones \\
\hline & & & 0 \\
& Con tratamiento & $5^{\circ} \mathrm{C}$ & 20 días \\
& térmico $\left(90^{\circ} \mathrm{C} / 60 \mathrm{~s}\right)$ & $-5^{\circ} \mathrm{C}$ & días \\
& & & 60 días \\
80 días & \\
& & & 0 \\
& & & 20 días \\
& & & 40 días \\
& Sin tratamiento & $-5^{\circ} \mathrm{C}$ & 60 días \\
& térmico & $-15^{\circ} \mathrm{C}$ & 80 días \\
\hline
\end{tabular}

Fuente: Elaboración propia (2019)

La ecuación matemática es la siguiente:

$Y_{i j k}=\mu+\alpha_{i}+\beta_{j}+(\alpha \beta)_{i j}+\varepsilon_{i j k}$

Donde:

$Y_{i j k}:$ Variable de respuesta de la k-ésima observación bajo la j-ésimo nivel de factor tiempo de almacenamiento sujeto a la i-ésimo nivel de tratamiento temperatura de almacenamiento.

$\mu \quad$ : Efecto de la media de la población a la cual pertenecen las observaciones.

$\alpha_{i}$ : Efecto de la i-ésimo nivel del factor temperatura de almacenamiento.

$\beta_{j}$ : Efecto del j-ésimo nivel del factor tiempo de almacenamiento.

$(\alpha \beta)_{i j}$ : Efecto interacción del i- ésimo nivel del factor temperatura, en el j- ésimo nivel del factor tiempo de almacenamiento.

$\varepsilon_{i j k}:$ Efecto del error experimental.

\section{Resultados y discusiones}

\begin{tabular}{|c|c|}
\hline \multicolumn{2}{|c|}{$\begin{array}{l}\text { Obtención de pulpa de copoazú } \\
\text { Tabla } 2 \\
\text { Rendimiento de copoazú. }\end{array}$} \\
\hline Producto & Rendimiento (\%) \\
\hline Cáscara & 49,43 \\
\hline Semilla & 17,71 \\
\hline Pulpa & 26,33 \\
\hline Placenta & 6,53 \\
\hline
\end{tabular}

El rendimiento de pulpa de copoazú obtenido en la investigación es de 26,3\%, este valor es inferior a lo reportado por Venturieri (1993) quien reporta un rendimiento de 38,5\% y Arizaleta et al., (2015) con 32,79\% en masa fresca. Es importante recalcar que en el presente estudio se consideró el porcentaje de la placenta por separado, la cual representa un 6,53\%.

\section{Evaluación fisicoquímica de la pulpa de copoazú}

Citar como: Cuse Quispe, J., Lima Pimentel, V. H. ., \& Cutipa Chávez, L. . . (2021). Cinética de la degradación y estabilidad del ácido ascórbico en la pulpa de copoazu (Theobroma grandiflorum). Revista Agrotecnológica Amazónica, 1(1), 4-19. https://doi.org/10.51252/raa.v1i1.113 


Tabla 3
\begin{tabular}{lr} 
Análisis fisicoquímico de la pulpa de copoazú \\
\hline \multicolumn{2}{c}{ Características Fisicoquímicas } \\
\hline Sólidos solubles ( ${ }^{\circ}$ Brix ) & Valor \\
pH & 11,33 \\
Acidez Titulable (exp. en \% Ác. Cítrico) & 3,18 \\
Ácido Ascórbico (mg/100 g) & 2,27 \\
\hline Fuente: Elaboración propia (2019). & 58,22 \\
\hline
\end{tabular}

En la Tabla 3 se puede apreciar que la pulpa de copoazú (Theobroma gradiflorum) es un producto con un alto contenido de ácido ascórbico $(58,22 \mathrm{mg} / 100 \mathrm{~g})$, este resultado se asemeja a lo reportado por Martin (2013), quien en su estudio encontró 56,16 mg ácido ascórbico/100 g. de muestra. De la misma manera el valor de sólidos solubles obtenidos experimentalmente se encuentra por debajo de lo reportado por Martin (2013) (18,59 ${ }^{\circ}$ Brix), pero estos se encuentran dentro del rango reportado por Villachica (1996), INIAP (2008) mencionado por Pérez (2015) con 10,80 y $11,17^{\circ}$ Brix; el valor de $\mathrm{pH}$ obtenido experimentalmente $(3,18)$ se encuentra dentro del rango obtenido por los autores mencionados antes y el valor obtenido experimentalmente de la acidez titulable (2,27\% ácido cítrico) se encuentra en similar rango según lo mencionado por Pérez (2015) y Martin (2013).

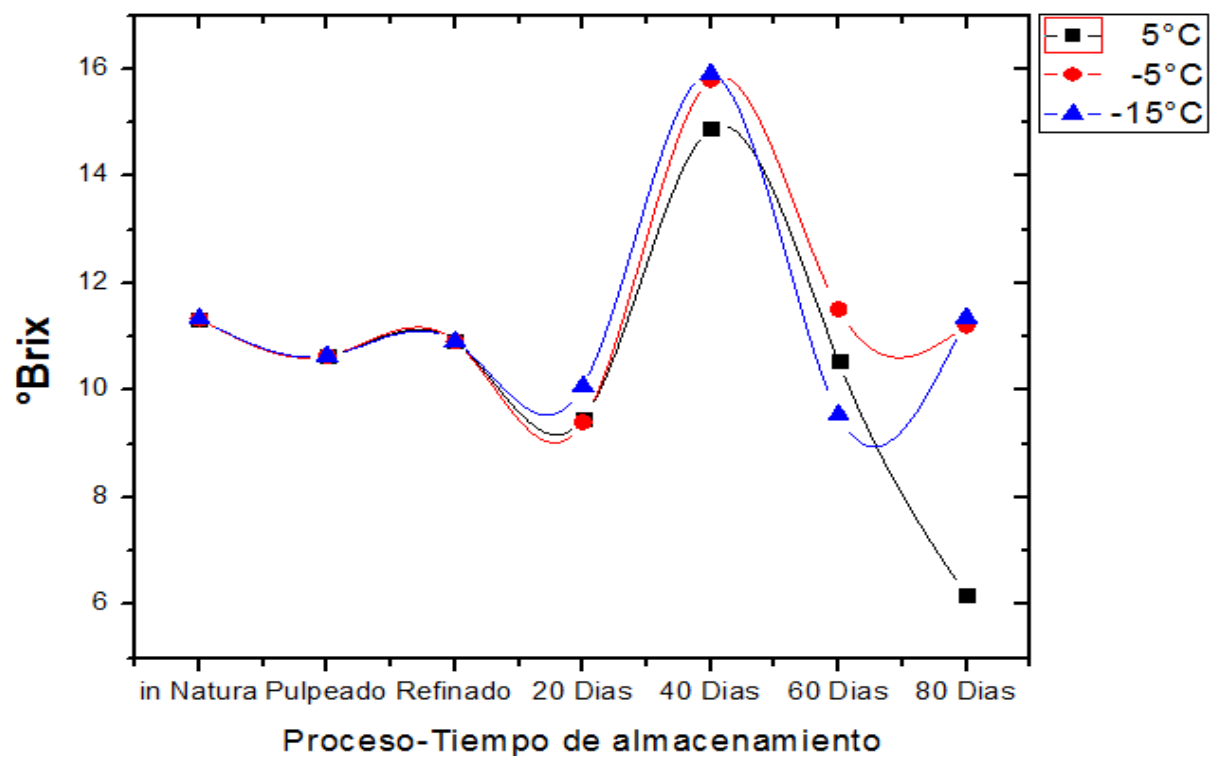

Figura 2: Comportamiento de ${ }^{\circ}$ Brix en la pulpa de copoazú sin tratamiento térmico durante el procesado y almacenamiento.

En la Figura 2 se aprecia como los procesos de pulpeado y refinado influyen mínimamente en el contenido de ${ }^{\circ}$ Brix, con una pérdida $0,69^{\circ}$ Brix en el proceso de pulpeado $\left(10,64{ }^{\circ} \mathrm{Brix}\right)$ y un ligero aumento de $0,27{ }^{\circ}$ Brix durante la etapa de refinado en referencia a la etapa anterior, según Castellano et al., (2016), a medida que la temperatura aumenta, la concentración de sólidos solubles se incrementa, esta concentración de azucares en los jugos se incrementa por efectos de la degradación de los polisacáridos de las membranas celulares.

Citar como: Cuse Quispe, J., Lima Pimentel, V. H. ., \& Cutipa Chávez, L. . (2021). Cinética de la degradación y estabilidad del ácido ascórbico en la pulpa de copoazu (Theobroma grandiflorum). Revista Agrotecnológica Amazónica, 1(1), 4-19. https://doi.org/10.51252/raa.v1i1.113 


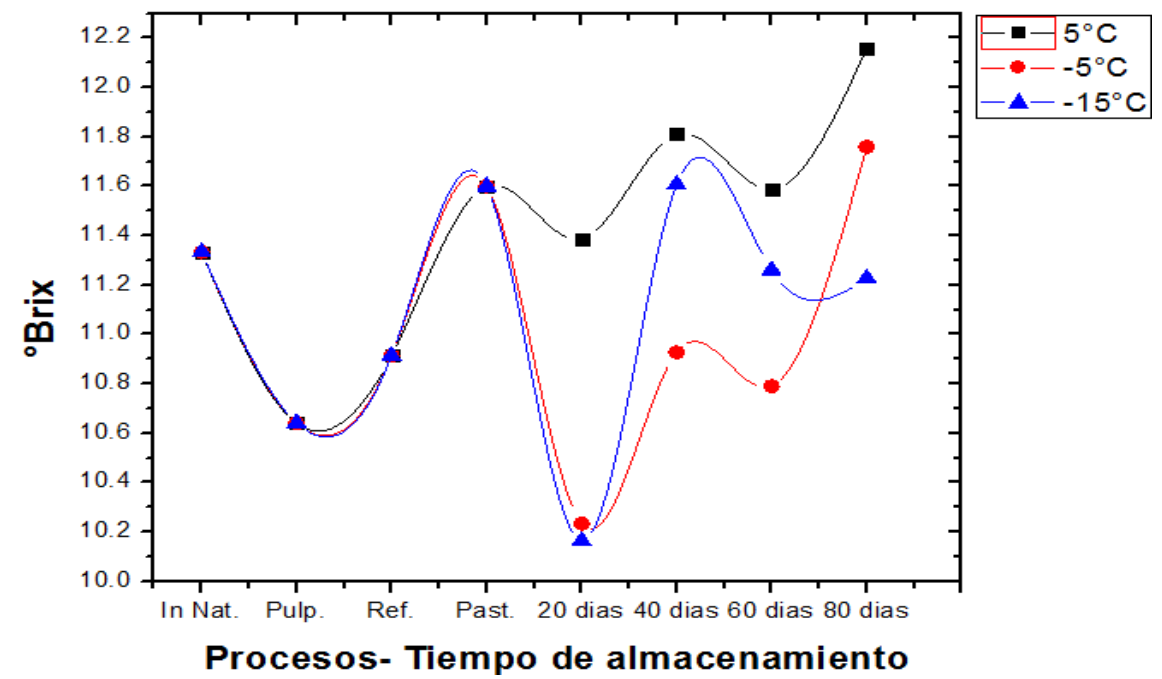

Figura 3: Comportamiento de ${ }^{\circ}$ Brix en la pulpa de copoazú pasteurizada durante el procesado y almacenamiento.

En la Figura 3 para la muestra de pulpa de copoazú con tratamiento térmico se puede apreciar un aumento de $0,69{ }^{\circ}$ Brix en referencia a la última etapa desarrollada, esto se explica que debido a proceso térmico aplicado se elimina agua a través de evaporación, logrando una mayor concentración de los sólidos solubles, reflejándose en un aumento en la lectura de ${ }^{\circ}$ Brix (Castellano et al, 2016).

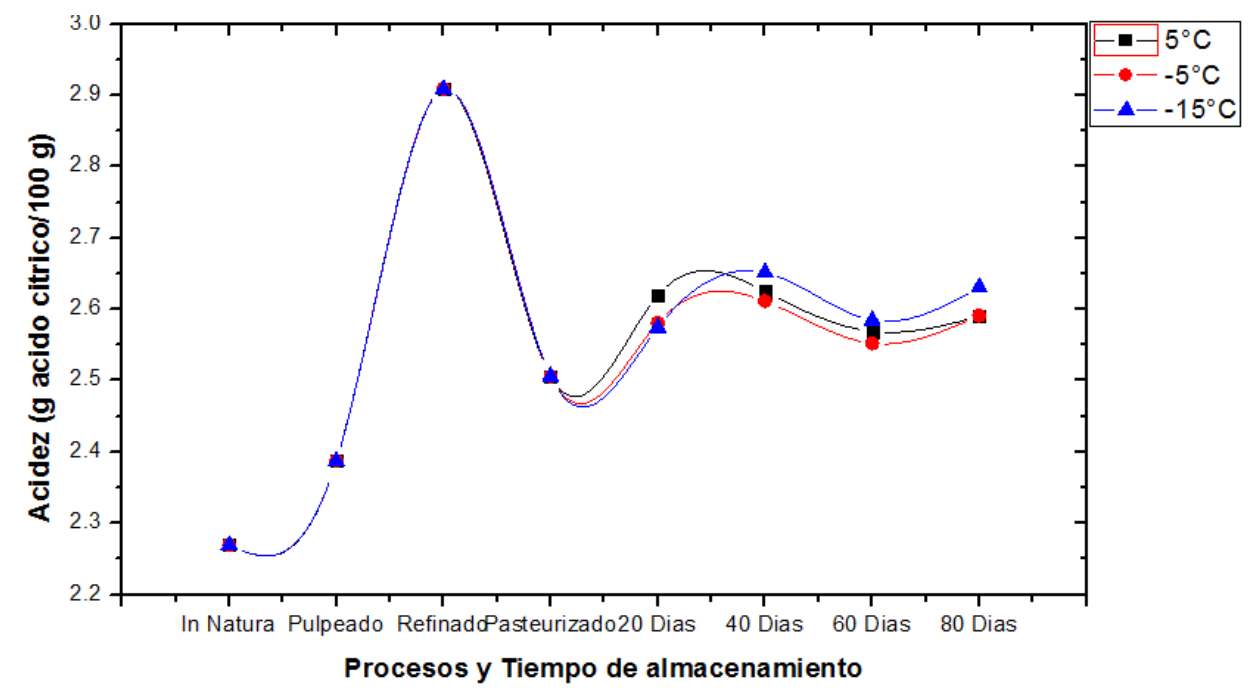

Figura 4: Comportamiento del ácido cítrico de la pulpa de copoazú pasteurizada durante el procesado y almacenamiento.

En la Figura 4 se observa que, durante la etapa del tratamiento térmico aplicado en la pulpa de copoazú, el valor de la acidez total disminuye de 2,91 a 2,51, esto referido a que al someter la pulpa a un proceso térmico se inactivan las enzimas presentes en la pulpa in natura, deteniendo el proceso de degradación y fermentación; según Azeredo (2012). 


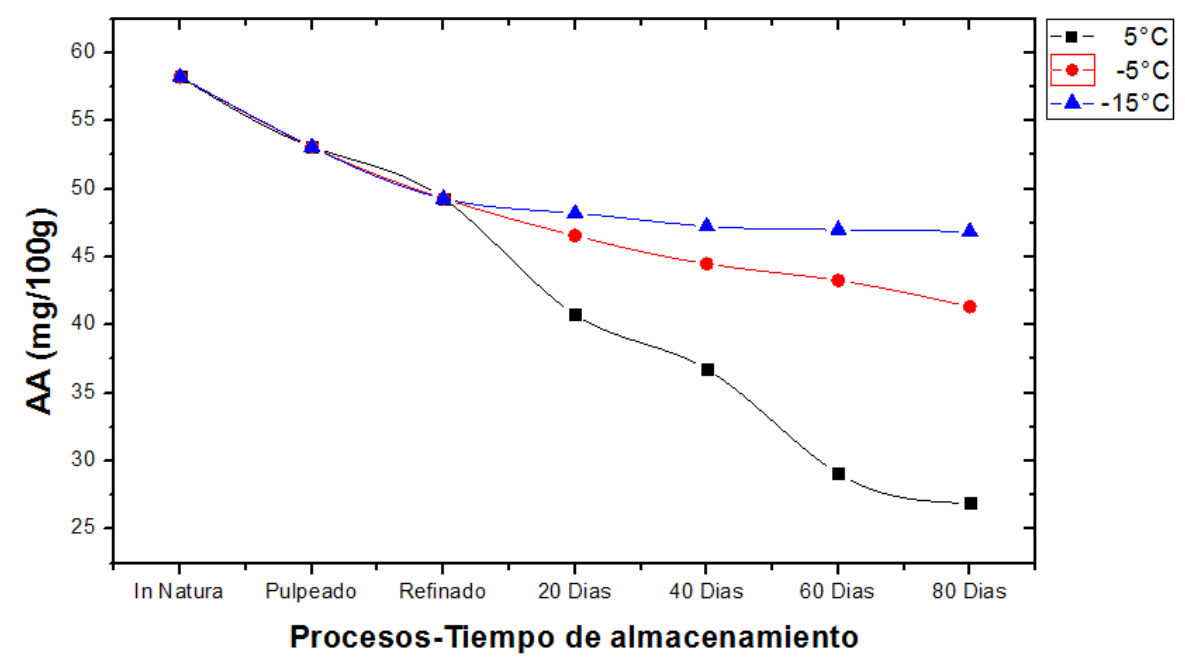

Figura 5: Comportamiento del Ácido ascórbico de la pulpa de copoazú sin tratamiento térmico durante el procesado y almacenamiento

En la Figura 5 se aprecia que las muestras sin tratamiento térmico almacenadas a $-5^{\circ} \mathrm{C}$ y $-15^{\circ} \mathrm{C}$ muestran un decremento leve de AA en el tiempo con valores finales de 41,35 y 46,86 mg/100g respectivamente; mientras que la muestra almacenada a $5^{\circ} \mathrm{C}$ es seriamente afectada en el tiempo con un valor final de $26,90 \mathrm{mg} / 100 \mathrm{~g}$.

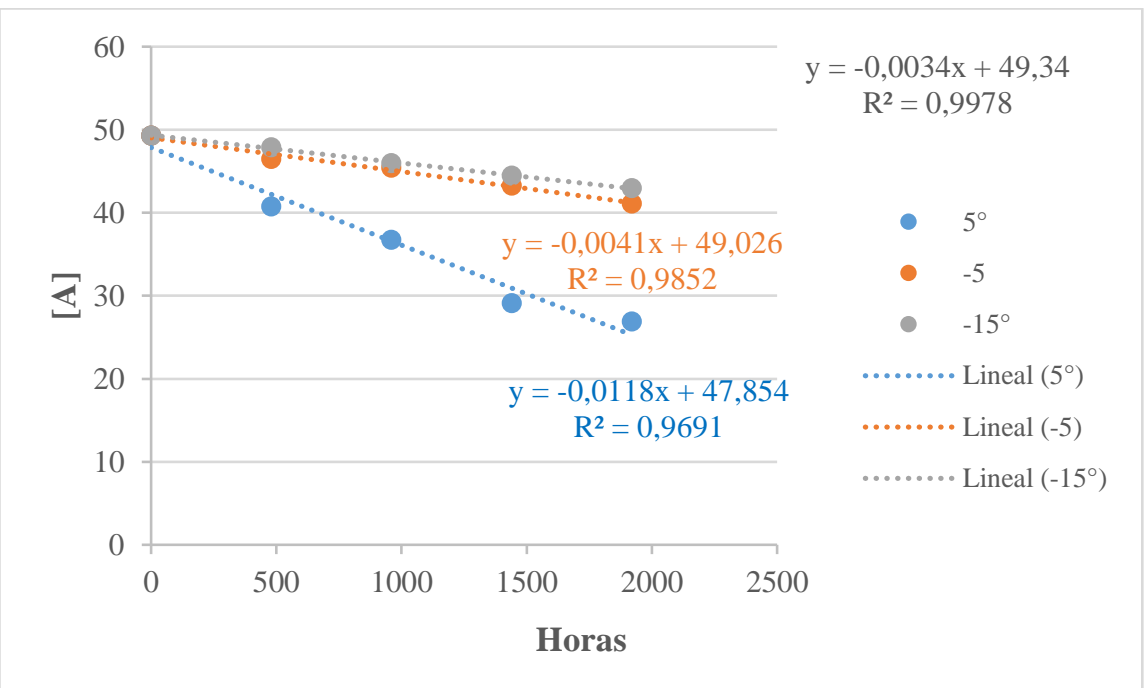

Figura 6: Cinética de degradación de ácido ascórbico en orden de reacción cero

En la Figura 6 se aprecian los valores de ácido ascórbico en función a la ecuación de orden cero, con un R2 igual a 0,$9691 ; 0,9852$ y 0,9987 para las temperaturas $5^{\circ} \mathrm{C},-5^{\circ} \mathrm{C}$ y $-15^{\circ} \mathrm{C}$ respectivamente. Siendo el mejor ajuste el reportado a una temperatura de $-15^{\circ} \mathrm{C}$.

Citar como: Cuse Quispe, J., Lima Pimentel, V. H. ., \& Cutipa Chávez, L. . (2021). Cinética de la degradación y estabilidad del ácido ascórbico en la pulpa de copoazu (Theobroma grandiflorum). Revista Agrotecnológica Amazónica, 1(1), 4-19. https://doi.org/10.51252/raa.v1i1.113 


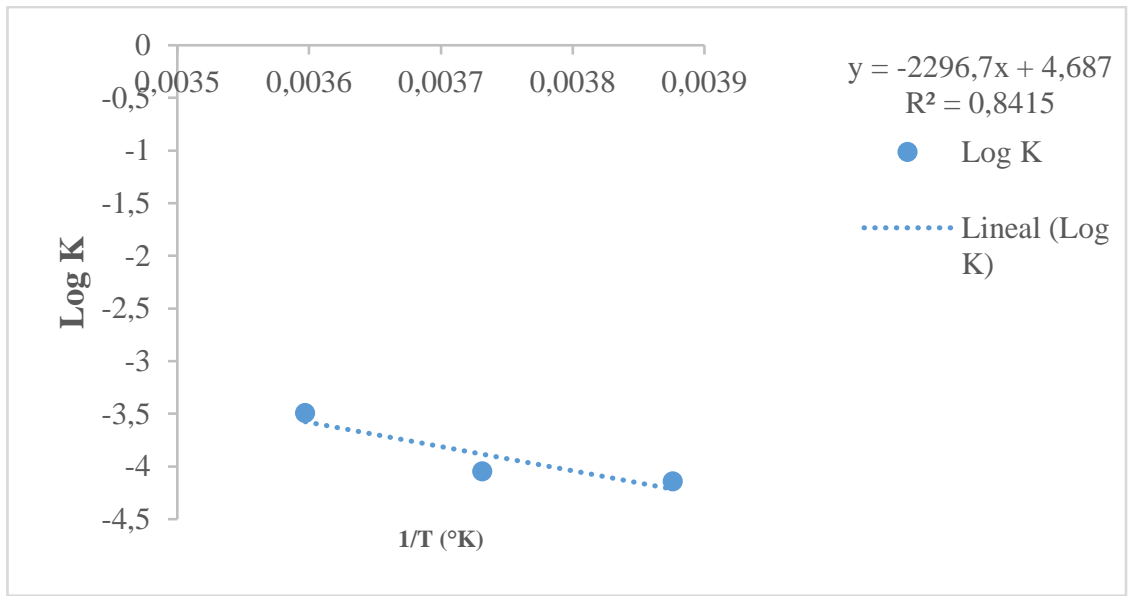

Figura 7: Gráfica de Arrhenius de la degradación de ácido ascórbico en la pulpa de copoazú sin tratamiento térmico

En la figura 7 se presenta para la pendiente un valor de 2296,7 con un $R 2=0,8415$, el valor del coeficiente de regresión resulto ser un poco menor que lo reportador por Vieira et al., (2000).

\section{Análisis estadístico}

Para la pulpa de copoazú con tratamiento térmico.

Tabla 4

Resumen estadístico para la degradación de AA en pulpa de copoazú con tratamiento térmico

\begin{tabular}{rrrrr}
\hline Grupos & Cuenta & Suma & Promedio & Varianza \\
\hline $5^{\circ} \mathrm{C}$ & 5 & 66,55 & 13,31 & 222,0835 \\
$-5^{\circ} \mathrm{C}$ & 5 & 184,29 & 36,858 & 1,33867 \\
$-15^{\circ} \mathrm{C}$ & 5 & 189,53 & 37,906 & 0,14498 \\
\hline
\end{tabular}

\section{Tabla 5}

Resultados ANOVA.

\begin{tabular}{lrrrrrr}
\hline \multicolumn{1}{c}{$\begin{array}{c}\text { Origen de las } \\
\text { variaciones }\end{array}$} & $\begin{array}{c}\text { Suma de } \\
\text { cuadrados }\end{array}$ & $\begin{array}{c}\text { Grados de } \\
\text { libertad }\end{array}$ & $\begin{array}{c}\text { Promedio de } \\
\text { los cuadrados }\end{array}$ & F & P valor & $\begin{array}{c}\text { Valor crítico } \\
\text { para F }\end{array}$ \\
\hline $\begin{array}{l}\text { Entre tratamientos } \\
\text { Dentro de los tra- }\end{array}$ & 1934,2830 & 2 & 967,14152 & 12,977 & 0.000998 & 3.88529 \\
tamientos & 894,2686 & 12 & 74,522383 & & & \\
Total & 2828,5516 & 14 & & & & \\
\hline
\end{tabular}

Se puede observar que existe diferencia estadísticamente significativa entre un tratamiento y otro con respecto al nivel de temperatura de almacenamiento de la pulpa de copoazú ( $\mathrm{P}$ valor $<0.05$ ), con un nivel de confianza del 95\%, lo que nos indica que los diferentes tratamientos influyen significativamente en al menos un tratamiento en el contenido de ácido ascórbico presente en la pulpa de copoazú tratada térmicamente.

\section{Conclusiones}

Se determinó las características físico químicas (AA, pH, Acidez titulable y ${ }^{\circ}$ Brix) de la pulpa de copoazú y su estabilidad durante las etapas de procesamiento y almacenamiento, logrando retener para la muestra pasteurizada a temperatura de almacenamiento de $-5^{\circ} \mathrm{C}$ y $-15^{\circ} \mathrm{C}$ durante

Citar como: Cuse Quispe, J., Lima Pimentel, V. H. ., \& Cutipa Chávez, L. . (2021). Cinética de la degradación y estabilidad del ácido ascórbico en la pulpa de copoazu (Theobroma grandiflorum). Revista Agrotecnológica Amazónica, 1(1), 4-19. https://doi.org/10.51252/raa.v1i1.113 
80 días un promedio de $62 \%$ de ácido ascórbico y para la muestra sin tratamiento térmico un promedio de $75 \%$ de ácido ascórbico. Mientras que para las muestras almacenadas a $5^{\circ} \mathrm{C}$ se retuvo $5,82 \%$ (tratado térmicamente) y 46,22\% (sin tratamiento térmico).

La velocidad de degradación del ácido ascórbico durante el almacenamiento de la pulpa de copoazú con tratamiento térmico es: $\mathrm{K} 5^{\circ} \mathrm{C}=0,0013$ (horas-1)., $\mathrm{K}-5^{\circ} \mathrm{C}=6,81 \mathrm{E}-05$ (horas-1), $\mathrm{K}$ $15^{\circ} \mathrm{C}=3,89 \mathrm{E}-5$ (horas-1) y para la muestra sin tratamiento térmico: $\mathrm{K} 5^{\circ} \mathrm{C}=0,0003$ (horas-1)., $\mathrm{K}-5^{\circ} \mathrm{C}=9,05 \mathrm{E}-05$ (horas-1), $\mathrm{K}-15^{\circ} \mathrm{C}=7,28 \mathrm{E}-5$ (horas-1). Se concluye que temperaturas inferiores a $-17^{\circ} \mathrm{C}$ preservan en mayor medida los valores de ácido ascórbico en la pulpa de copoazú pasteurizada y de igual manera con las muestras sin tratamiento térmico.

La estabilidad del ácido ascórbico en la pulpa de copoazú en función de las constantes de degradación cinética (K horas-1) a diferentes temperaturas es: Para la pulpa sin tratamiento térmico a $5^{\circ} \mathrm{C}=4,34$ meses, $-5^{\circ} \mathrm{C}=15,46$ meses y $-15^{\circ} \mathrm{C}=19,21$ meses; para la pulpa con tratamiento térmico a $5^{\circ} \mathrm{C}=0,8$ meses, $-5^{\circ} \mathrm{C} 15,33$ meses y $-15^{\circ} \mathrm{C}=26,8$ meses.

\section{Referencias bibliográficas}

ARIZALETA, M., ÁLVAREZ, A. y PÉREZ, M., 2015. Caracterización De Frutos Y Efecto De Temperatura De Almacenamiento Sobre Calidad De Pulpa De Copoazú. Revista Unellez de ... [en línea], Disponible en: http://150.187.216.91/revistas/index.php/rucyt/article/view/302.

AZEREDO, H.M.C. De, 2012. Fundamentos de estabilidade de alimentos. Fundamentos de Estabilidade de Alimentos, EMBRAPA [en línea], pp. 326. ISSN 0031-9023. Disponible en:

http://scholar.google.com/scholar?hl=en\&btnG=Search\&q=intitle:Fundamentos+de+estabi lidade+de+alimentos\#0.

BADUI, S., 2013. Química Alimentos. S.1.: s.n. ISBN 9786073215084.

BENASSI, M. T.; ANTUNES, A.J., 1988. A comparison of metaphosphoric and oxalic acids as extractant solutions for the determination of vitamin $\mathrm{C}$ in selected vegetables. Arquivos de Biologia e Tecnologia, vol. 31, pp. 507-513.

CASTELlANO, G., RAMÍREZ, R., SINDONI V., M.J., HIDALGO L., P.R., BURGOS, M.E., MARÍN R, C., NÚÑEZ-CASTELLANO, K. y MARTÍNEZ, L., 2016. EFECTO DE LA TEMPERATURA DE ALMACENAJE SOBRE LAS CARACTERÍSTICAS

ORGANOLÉPTICAS DE FRUTOS DE LIMÓN PERSA (Citrus latifolia Tanaka). Revista Iberoamericana de Tecnología Postcosecha, ISSN 1665-0204.

GONZALES, M., 2010. Conservación de mora, uvilla y frutilla mediante la utilización del 
aceite esencial de canela (cinnamomum zeynalicum). S.l.: Riobamba. Ecuador. Escuela Superior Politécnica de Chimborazo.

FIETZ, V.R. y SALGADO, M.S., 1999. Efeito da pectina e da celulose nos niveis séricos de colesterol e triglicerídos em ratos hiperlipedémicos. Ciencia E Tecnologia De Alimentos, vol. 19, pp. 318-321.

LIM, T.K., 2012. Edible Medicinal And-Nom-Medicinal Plant. Volumen 3. S.l.: s.n.

MARTIN, L.G.P., 2013. Estudo da secagem da polpa de cupuaçu por atomização. , pp. 110.

MARTINS, V.B., 2008. PERFIL SENSORIAL DE SUCO TROPICAL DE CUPUAÇU

(Theobroma grandiflorum Schum). S.l.: s.n.

MATOS, A. y CHUQUILÍN, E.F., 2010. Estudio de la Influencia de la Concentración en la Retención de Vitamina C en Jugo Fresco y Concentrado de Carambola (Averrhoa carambola L.). Investigación en Ciencia y Tecnología de Alimentos [en línea], vol. 1, no. 2218-3310, pp. 36-42. ISSN 2218-3310. Disponible en: http://investigacion.upeu.edu.pe/images/Journal/RVCITAL01/Chuquilin-Eddy.pdf.

MELGAREJO, M.L., HERNÁNDEZ, M.S., BARRERA, J.A. y CARRILLO, M., 2006. Oferta y potencialidades de un Banco de Germoplasma del Genero Theobroma en el Enriquecimiento de los sistemas productivos de la Región Amazónica. 1. Bogotá D.C.: Editorial Scripto Ltda. ISBN 9589795080, 9789589795088.

MENDOZA, S. Y QUIÑONES, R., 2005. Evaluación del proceso de deshidratación del camucamu (Myrciariadubia H. B. K. Mc Vaugle) mediante secador de lecho fluidizado y obtención de los parámetros cinéticos de degradación térmica del ácido ascórbico. S.1.: Universidad Nacional del Centro del Perú.

PÉREZ, P., 2015. COMPORTAMIENTO REOLÓGICO DE LA PULPA TAMIZADA DE 4 COPOAZÚ (Theobroma grandiflorum). S.1.: Universidad Nacional Amazónica de Madre de Dios.

SANTOS, G. M., MAIA G. A., SOUSA, P. M., FIGUEIREDO, R. W., COSTA, J.M.C. y FONSECA, A.V. V, 2010. Antioxidant activity and correlations with bioactive components from commercial products of cupuaçu. Ciencia Rural, vol. 40, pp. 1636-1642.

SILVA, F.M., SIMS, C., BALABAN, M.O., SILVA, C.L.M. y O'KEEFE, S., 2000. Kinetics of flavour and aroma changes in thermally processed cupuacu (Theobroma grandiflorum) pulp. Journal of the Science of Food and Agriculture, vol. 80, no. 6, pp. 783-787. ISSN 00225142. DOI 10.1002/(SICI)1097-0010(20000501)80:6<783::AID-JSFA622>3.0.CO;2R.

Citar como: Cuse Quispe, J., Lima Pimentel, V. H. ., \& Cutipa Chávez, L. . (2021). Cinética de la degradación y estabilidad del ácido ascórbico en la pulpa de copoazu (Theobroma grandiflorum). Revista Agrotecnológica Amazónica, 1(1), 4-19. https://doi.org/10.51252/raa.v111.113 
TAVARES, J.C., GÓIS, V.D. DE, NETO, F.B. y OLIVEIRA, M.J.A. DE, 1998. Métodos de congelamento na conservação da acerola (Mapighia emarginata DC.). . S.l.:

VELEZ, G.A., 1991. Los Frutales amazónicos cultivados por las comunidades indígenas de la region del Medio Caquetá (Amazonia Colombiana). Revista Colombiana Amazónica, vol. 5, pp. 163-193.

VENTURIERI, G.A., 1993. Cupuaçu: A espécie, sua cultura, usos, e processamento. 1993. S.1.: Clube do cupu.

VIEIRA, M.C., TEIXEIRA, A.A. y SILVA, C.L.M., 2000. Mathematical modeling of the thermal degradation kinetics of vitamin $\mathrm{C}$ in cupuaçu (Theobroma grandiflorum) nectar. Journal of Food Engineering, vol. 43, no. 1, pp. 1-7. ISSN 02608774. DOI 10.1016/S0260-8774(99)00121-1.

\section{Agradecimientos}

A la Universidad Nacional Amazónica De Madre De Dios por habernos facilitado los laboratorios y plantas pilotos para los respectivos análisis. 


\section{Anexos}

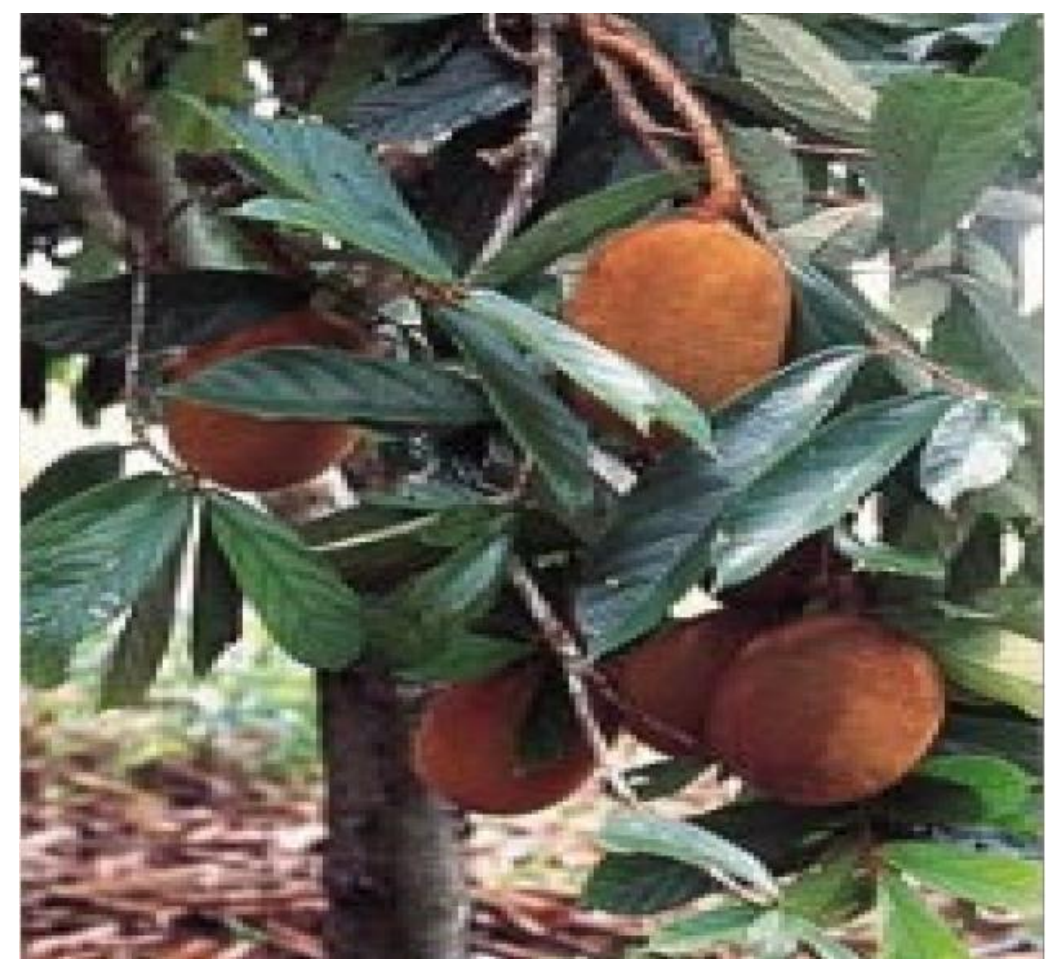

Árbol de copoazú (Theobroma grandiflorum)

Fuente: Ferreira (2008)

\section{Conflicto de intereses.}

Declaramos que no existen conflictos de interés.

\section{Contribuciones de los autores.}

-El Bach. Víctor Hugo lima Pimentel, se encargó de la parte ejecutora del proyecto

-La Dra. Lastenia Cutipa Chávez, tuvo a su cargo la parte del análisis químico en el laboratorio y la planta piloto, así como la asesoría.

-El Dr. Jaime Cuse Quispe, tuvo participación en el análisis estadístico, así como en la redacción y la parte metodológica.

Citar como: Cuse Quispe, J., Lima Pimentel, V. H. ., \& Cutipa Chávez, L. . . (2021). Cinética de la degradación y estabilidad del ácido ascórbico en la pulpa de copoazu (Theobroma grandiflorum). Revista Agrotecnológica Amazónica, 1(1), 4-19. https://doi.org/10.51252/raa.v1i1.113 